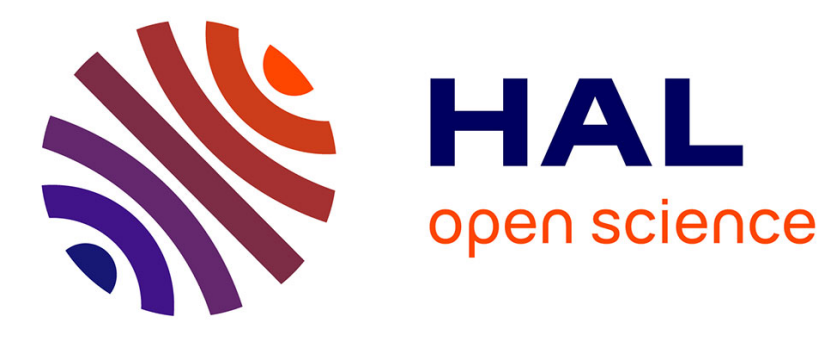

\title{
Le théâtre chiite au Liban, entre rituel et spectacle Sabrina Mervin
}

\section{To cite this version:}

Sabrina Mervin. Le théâtre chiite au Liban, entre rituel et spectacle . Franck Mermier, Nicolas Puig. Itinéraires esthétiques et scènes culturelles, IFPO, pp.57-75 2007, 9782351592687. 10.4000/books.ifpo.517 . halshs-01859233

\section{HAL Id: halshs-01859233 \\ https://shs.hal.science/halshs-01859233}

Submitted on 27 Aug 2018

HAL is a multi-disciplinary open access archive for the deposit and dissemination of scientific research documents, whether they are published or not. The documents may come from teaching and research institutions in France or abroad, or from public or private research centers.
L'archive ouverte pluridisciplinaire HAL, est destinée au dépôt et à la diffusion de documents scientifiques de niveau recherche, publiés ou non, émanant des établissements d'enseignement et de recherche français ou étrangers, des laboratoires publics ou privés. 


\section{(2) OpenEdition Books}
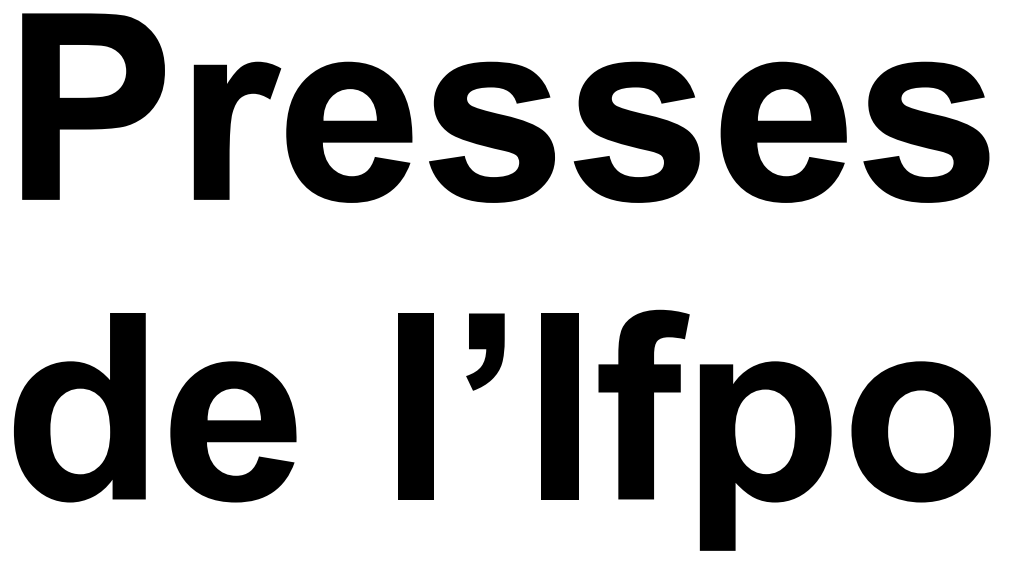

Itinéraires esthétiques et scènes culturelles au Proche-Orient $\mid$ Nicolas Puig, Franck Mermier

\section{Le théâtre chiite au}

Liban, entre rituel

et spectacle

Sabrina Mervin

p. $57-75$

\section{Entrées d'index}

Mots clés :

Ce site utilise des cookies et collecte des informations personnelles vous concernant.

Pour plus de précisions, nous vous invitons à consulter notre politique de confidentialité

(mise à jour le 25 juin 2018).

En poursuivant votre navigation, vous acceptez l'utilisation des cookies. 
1 Ce théâtre est d'abord un rituel qui commémore la passion de Husayn, petit-fils du prophète Muhammad et troisième imam pour les chiites duodécimains. Il s'agit de se lamenter sur son martyre et sur les malheurs de la famille sacrée, les ahl al-bayt, car cela est recommandé dans les doctrines chiites : les larmes pour Husayn mènent le fidèle au paradis et le rapprochent de Dieu, qu'il pleure, qu'il fasse mine de pleurer ou bien qu'il fasse pleurer autrui. Ce rituel vise aussi à transmettre une histoire sacrée dont on tire des enseignements et des valeurs morales en exaltant les qualités et les attitudes de personnages qui sont des références suprêmes, infaillibles (Husayn), ou bien des modèles à imiter (sa sœur Zaynab), ou encore des repoussoirs (Yazîd et son armée).

2 Husayn, fils de 'Alî et de Fâtima, est mort en 680 lors de la bataille de Karbala. Une histoire anthropologique de l'événement reste à faire ; de ce point de vue, il peut être analysé comme une lutte de pouvoir entre deux clans, les hachémites et les banû Sufyân. Cependant, il fonctionne comme un mythe fondateur du chiisme qui soude la communauté et forge sa mémoire collective autour d'une une historiographie qui rapporte, en prose et, surtout, en vers, chaque épisode de l'épopée avec moult détails. Voici un résumé de cette épopée, telle qu'elle est rapportée aujourd'hui.

3 Le premier imam, 'Alî, fut assassiné en 660, alors que le

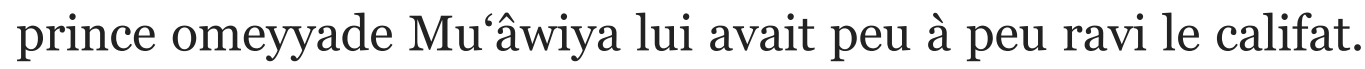
Après sa mort, son fils aîné Hasan conclut un pacte avec Mu'âwiya, et renonça ainsi au pouvoir. Lorsque Mu'âwiya mourut à son tour, il passa la succession à son fils Yazîd. Or, Hasan étant décédé entre-temps, son frère cadet Husayn, qui était devenu le chef de la famille, ne s'estimait plus tenu par le pacte. Il refusa de prêter allégeance au nouveau calife. Les 
assiégèrent son camp et lui coupèrent l'accès aux eaux de l'Euphrate. Pressions et négociations n'aboutirent à aucun accord : Husayn persista à refuser de prêter allégeance au calife Yazîd. À l'aube du dixième jour du mois de muharram, celui de Achoura, on livra bataille. Les partisans de Husayn, des membres de sa famille, tombèrent un à un ; le dernier à périr fut son demi-frère 'Abbâs. Puis ce fut le tour de Husayn. Les têtes des vaincus furent brandies sur des lances par les soldats omeyyades, les femmes et les enfants furent capturés et emmenés à Kûfa, puis à Damas. Là, ils comparurent à la cour du calife qui les fit emprisonner, avant de les libérer et de les laisser regagner Médine. Les habitants de Kûfa, quant à eux, n'étaient pas intervenus pour sauver Husayn. Ceux qui tentèrent de le venger par la suite furent tués à leur tour.

4 Selon les doctrines chiites, le martyre de Husayn doit permettre l'accomplissement de son imamat et la revivification de la religion fondée par son grand-père. Par les rituels observés chaque année, les fidèles renouvellent leur allégeance et leur dévotion à Husayn et aux imams ; ils manifestent amour et compassion pour les gens de la Maison $\mathrm{du}$ prophète (ahl al-bayt). Ce sont donc les spécificités dogmatiques du chiisme, notamment la théorie de l'imamat, qui sont ainsi affirmées, réitérées. En outre, le martyre de Husayn, c'est le paradigme du sacrifice de soi, de la lutte entre le bien et le mal, du combat contre l'oppression et l'injustice, de la révolution (thawra) comme nécessité, " la victoire du sang contre l'épée », un idéal à atteindre" Il fonctionne comme un événement fondateur que les fidèles reproduisent dans des célébrations où le temps et l'espace sont abolis, selon le slogan : " chaque jour, c'est Achoura et chaque terre, c'est Karbala » (kull yawm 'âshûrâ' wa kull ard Karbalâ'). L'événement est intériorisé par les fidèles, qui

Ce site utilise des cookies et collecte des informations personnelles vous concernant. Pour plus de précisions, nous vous invitons à consulter notre politique de confidentialité (mise à jour le 25 juin 2018).

En poursuivant votre navigation, vous acceptez l'utilisation des cookies. 
5 «Quel que soit le moyen mis en ouvre, il s'agit d'ouvrir et de rouvrir périodiquement la source des pleurs... », écrivait Virolleaud sur le théâtre persan². Pour ce faire, une abondante littérature s'est constituée, notamment un énorme corpus de poèmes en arabe classique comme en dialecte (surtout irakien), et quatre types de rituels se sont mis en place au fil des siècles ${ }^{3}$. Les fidèles rendent des visites pieuses aux mausolées des imams ou des ahl al-bayt, particulièrement à celui de Husayn, à Karbala, le jour de Achoura, puis le «quarantième » jour après la date du martyre (al-arbaîn) ; ceux qui ne peuvent s'y déplacer effectuent une visite symbolique, par la prière. Dès le début du mois de muharram, et jusqu'à l'arba'în, des séances de déploration (majâlis husayniyya) sont organisées dans des lieux privés ou publics (les husayniyya, lieux de culte ad hoc, ou les écoles religieuses) : un ou une récitant(e) y égrène, jour après jour, les épisodes de la bataille de Karbala, faisant à la fois le narrateur et les différents personnages, et passant de la prose à la poésie, de l'arabe soutenu au dialecte. Plus l'on avance vers le jour de Achoura, plus la ferveur et l'émotion montent. Des processions publiques (mawâkib husayniyya) rassemblent les fidèles, regroupés par affinités, affiliation politique, secteur géographique, qui défilent en se frappant la poitrine (latm). Certains pratiquent des rites de mortification, notamment le tatbîr, faisant couler le sang du haut de leur tête sur un tissu blanc dont ils sont revêtus, qui symbolise le linceul de Husayn ${ }^{4}$. Tout l'espace est transformé par les banderoles noires avec des slogans brodés, les affiches avec des dessins de scènes de la bataille, les drapeaux, la foule qui se presse, vêtue de noir, dans un environnement sonore qui s'emplit des voix des récitants de majlis diffusées par des haut-parleurs. C'est dans cette atmosphère particulière, oscillant entre la célébration de

Ce site utilise des cookies et collecte des informations personnelles vous concernant. Pour plus de précisions, nous vous invitons à consulter notre politique de confidentialité (mise à jour le 25 juin 2018).

En poursuivant votre navigation, vous acceptez l'utilisation des cookies. 
imams, celle du prophète et de Fâtima, ainsi que des sujets bibliques, des célébrations de saints locaux, etc. ${ }^{5}$. Certes, l'éventail est plus restreint au Liban, où ce théâtre a été en quelque sorte « importé » à la fin de l'Empire ottoman. On ne l'appelle pas ta'ziyeh, comme en persan, mais shabîh ou tashbîh, qui furent les premiers termes employés. Aujourd'hui, ils sont, dans la langue courante, remplacés par des expressions servant plus largement à désigner des représentations théâtrales (tamthîliyya, masrahiyya). En outre, des expressions sont créées pour désigner de nouvelles formes de représentations qui s'inscrivent dans ce théâtre rituel ('amal mashhadî, mashhadiyya 'âshurâ'iyya ${ }^{6}$ ).

7 Le jour de Achoura, des représentations de la bataille de Karbala et du martyre de Husayn sont jouées dans la ville de Nabatiyyeh, ainsi que dans des villages comme Majdal Silm, Jibchit, Zrariyyeh et Kafr Kila. Ensuite, d'autres pièces sont montées, autour du jour de l'arba‘în,notamment Le cortège des captives (Mawkib al-sabayâ) à Kafr Kila et al-Kharâ'ib, ou bien Les têtes (al-ru'ûs) à Jibchit. Elles sont issues du corpus existant en Iran, que l'on retrouve dans d'autres endroits où des chiites célèbrent Achoura, notamment en Turquie. De nouveaux types de représentation ont été créés récemment, dans le but de changer, de moderniser la forme, si ce n'est le contenu ; on en présentera ici deux exemples. Enfin, pendant toute la durée des célébrations, se mettent en place ce qu'on appelle des " panoramas », plus ou moins élaborés : ce sont, en fait, des tableaux de la bataille de Karbala reconstitués avec des mannequins, présentés avec un montage sonore et un arrangement de lumières.

Avant la représentation du Cortège des captives, un récitant raconte les malheurs de Husayn. Kafr Kila, 2005 .

Ce site utilise des cookies et collecte des informations personnelles vous concernant. Pour plus de précisions, nous vous invitons à consulter notre politique de confidentialité (mise à jour le 25 juin 2018).

En poursuivant votre navigation, vous acceptez l'utilisation des cookies. 


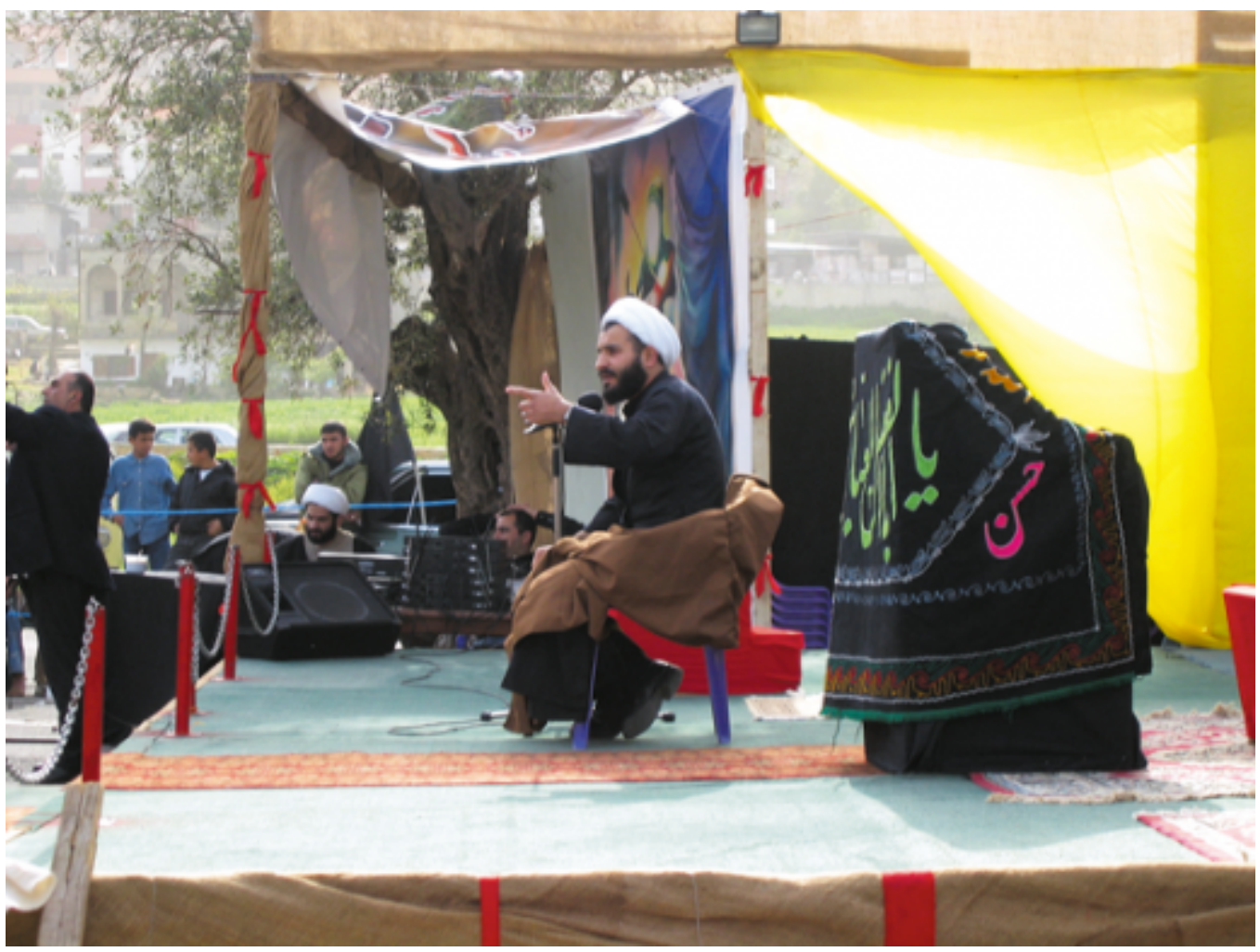

Photo S. Mervin.

\section{L’implantation du théâtre persan au Liban}

8 Le théâtre rituel en Iran a une histoire riche qui a fait l'objet d'ouvrages et de travaux scientifiques ${ }^{7}$. Il s'est formé en Iran au cours du $\mathrm{XvIII}^{\mathrm{e}}$ siècle et s'est organisé peu à peu sous le patronage des princes avec, notamment, la construction de théâtres en dur (tekiyeh ${ }^{8}$ ). On dispose déjà, sur cette période, de témoignages de voyageurs et de diplomates qui en donnent des descriptions ; d'autres, plus abouties, ont été fournies au XIx ${ }^{\mathrm{e}}$ siècle par Gobineau (1865), puis Arnold qui, le premier, compara la ta'ziyeh à la passion du Christ médiévale. Des textes de pièces ont été collectés, certains traduits. En 1970, Peter Brook s'y est intéressé. Depuis, ce théâtre a été mieux étudié, notamment par Peter Chelkowski ; il a été reconnu par l'Unesco et des troupes iraniennes sont allées jouer à Paris, à Parme et à New York ${ }^{9}$.

Ce site utilise des cookies et collecte des informations personnelles vous concernant. Pour plus de précisions, nous vous invitons à consulter notre politique de confidentialité (mise à jour le 25 juin 2018).

En poursuivant votre navigation, vous acceptez l'utilisation des cookies. 
l'objet «Hezbollah », avec une approche politiste qui ne privilégie pas les aspects culturels de son action.

9 Ce théâtre a été implanté dans l'actuel Liban-Sud, ainsi qu'à Damas, à la fin du $\mathrm{XIx}^{\mathrm{e}}$ siècle, par des Iraniens qui $\mathrm{y}$ résidaient. Autour de 1910, il s'est arabisé (puisqu’il était en persan) et, peu à peu, il s'est ancré dans la culture locale ${ }^{11}$. Cet ancrage se manifeste de différentes manières, dans les thèmes qui sont choisis comme dans leur mise en scène. Ainsi, dans les pièces iraniennes retraçant le drame de Karbala, un acteur vêtu d'un déguisement incarne le « personnage » du lion qui vient se lamenter sur le corps de Husayn, après sa mort. Le lion, qui symbolise 'Alî, « Lion de Dieu », est très présent dans la culture persane. Or, il a disparu des pièces libanaises : la greffe de cet élément important des représentations iraniennes n'a pas pris. En revanche, on retrouve le personnage d'al-Hurr ${ }^{12}$ dans toutes les pièces sur le drame de Karbala, ce qui n'est pas étonnant, puisqu'une famille du Liban-Sud se réclame de son ascendance. Seules les pièces principales, les plus connues, se sont implantées de manière pérenne au Liban. Toutefois, rien n'est figé : un nouvel emprunt peut se produire, une influence irakienne ou iranienne se faire sentir, et les textes sont retravaillés chaque année, certains étant plus labiles que d'autres.

10 Pour ce qui est de la scénographie, dans les pièces persanes, des musiciens jouent sur la scène, ou à côté ; ce n'est pas le cas au Liban. En Iran, la scène est au milieu des spectateurs, et souvent ronde ; au Liban, les spectateurs lui font face. En outre, le recours au symbolisme est plus développé en Iran où, par exemple, l'Euphrate est symbolisé par une bassine d'eau. Il en découle un dépouillement de ce théâtre, qui lui donne un aspect moderne. Au Liban, le désert de Karbala et le fleuve sont représentés sur un décor peint et c'est surtout

Ce site utilise des cookies et collecte des informations personnelles vous concernant. Pour plus de précisions, nous vous invitons à consulter notre politique de confidentialité (mise à jour le 25 juin 2018).

En poursuivant votre navigation, vous acceptez l'utilisation des cookies. 
Le rideau de scène du Cortège des captives. Kafr Kila, 2005.

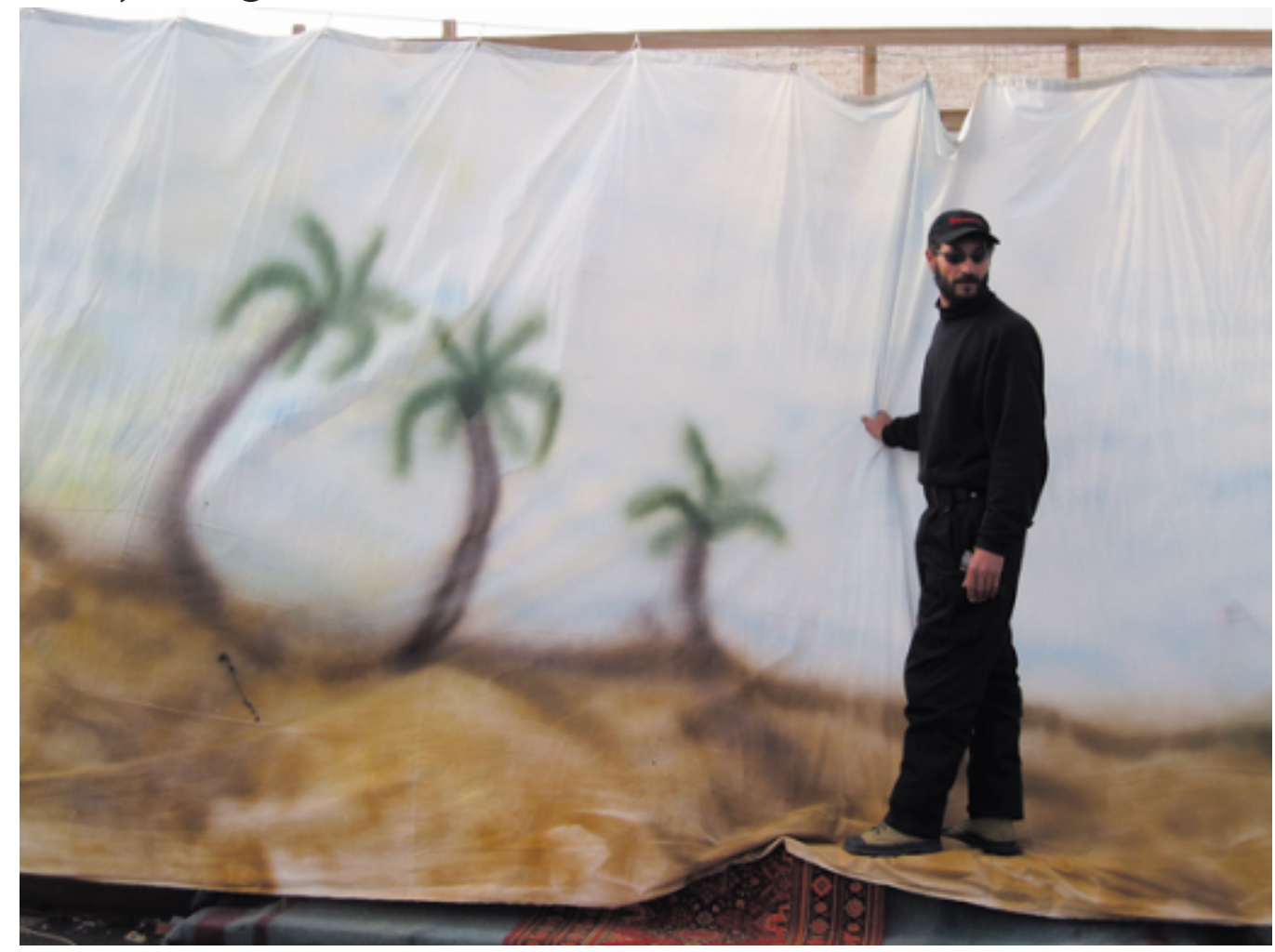

Photo S. Mervin.

11 La représentation de la bataille de Karbala s'est développée à Nabatiyyeh dans les années 1930, grâce au soutien du responsable religieux local, lui-même patronné par les notables et par la bourgeoisie commerçante de la ville. Un premier texte fut consigné, un « lieu scénique » fut délimité, sur l'aire à battre située au centre de la ville, les rôles furent distribués, et, peu à peu, « la » pièce de Nabatiyyeh se mit en place, chaque année renouvelée. Si son histoire a été écrite dans des travaux de recherche, à partir de sources orales, celle des petites pièces de village reste à faire.

\section{Un théâtre sacré et populaire}

12 Pour caractériser ce théâtre, il faut rappeler qu'il reste lié aux rituels dont il dérive et qui l'accompagnent, à savoir les 
aujourd'hui, seul un chameau participe encore à la procession qui amène les personnages sur scène. En revanche, certains personnages de la pièce sont à cheval et des cavalcades font partie intrinsèque de la représentation. Dans les villages, les chameaux ont disparu, et, bien souvent, il ne reste qu'un seul cheval.

\section{Cavalcades pour la représentation de la bataille de Karbala. Nabatiyyeh, 2005.}

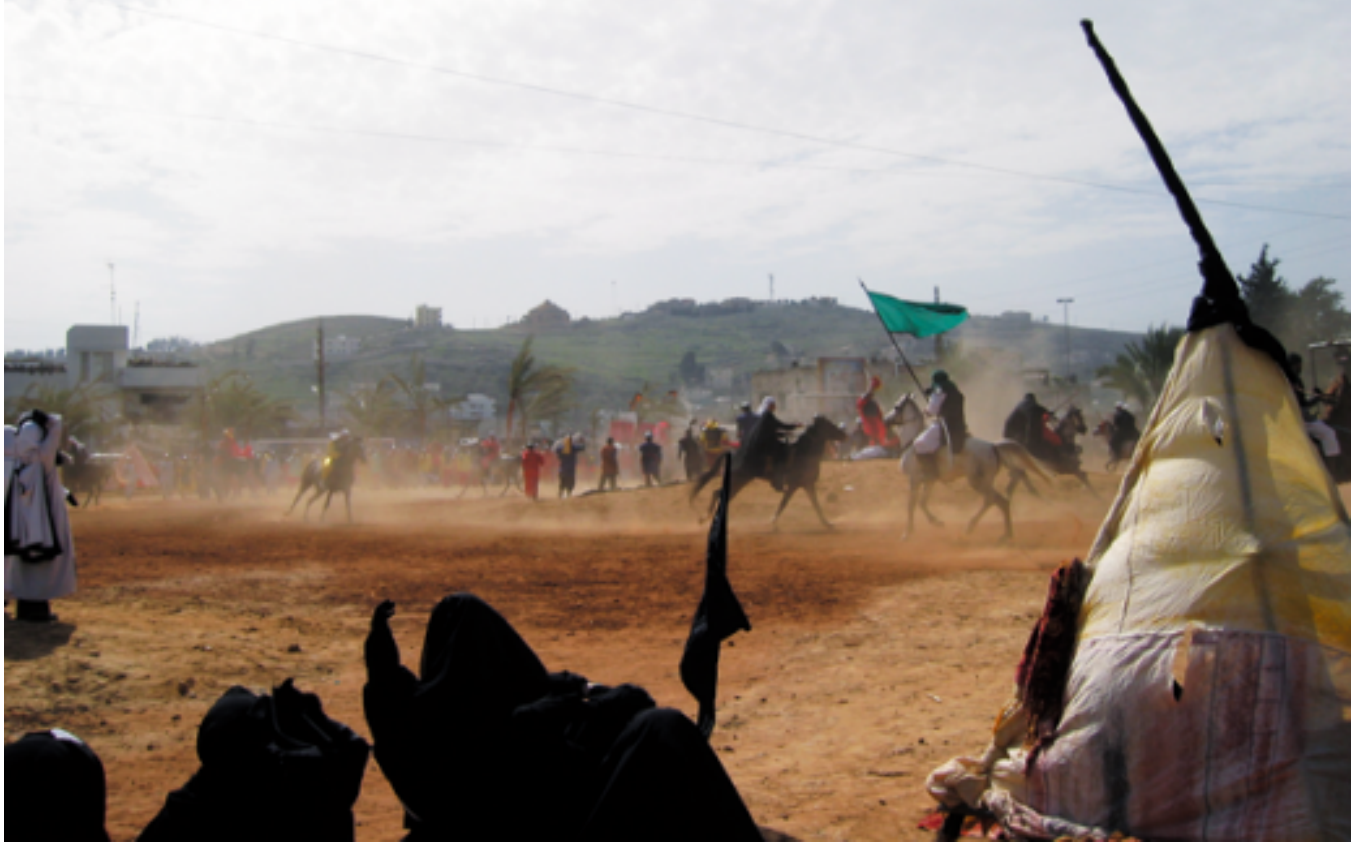

Photo S. Mervin.

13 Les représentations se déroulant souvent en plein air, certaines troupes ont recours à des micros-cravates pour amplifier les voix des acteurs... c'est là un progrès technique puisqu'il y a quelques années, ceux-ci se passaient un gros micro, de main en main ${ }^{15}$. D'autres ont opté pour une technique différente : la pièce est préalablement enregistrée, avec un montage mêlant les paroles des acteurs, les bruitages et les chants, et ce son « off » est diffusé pendant la

Ce site utilise des cookies et collecte des informations personnelles vous concernant. Pour plus de précisions, nous vous invitons à consulter notre politique de confidentialité (mise à jour le 25 juin 2018).

En poursuivant votre navigation, vous acceptez l'utilisation des cookies. 
14 Ce théâtre émane de groupes qui se constituent pour l'occasion, comme c'est le cas à Kafr Kila et dans d'autres villages dont les troupes sont locales, hétéroclites dans leur composition sociale et politique. Jouer ou participer à l'organisation des représentations est alors une œuvre pie, dont on n'attend pas de rémunération mondaine, mais une rétribution (ajr) dans l'au-delà ${ }^{16}$. D'autres troupes se rattachent à un groupe constitué, comme les scouts du mouvement Amal, ou bien, dans le cas du Hezbollah, elles sont formées par un organe du parti chargé de monter la pièce. Dans la plupart des cas, les acteurs ne sont pas rétribués.

15 Ce théâtre est financé de différentes manières. Dans les villages, les acteurs eux-mêmes, souvent, se procurent leurs costumes, et les participants à l'organisation aident à la fourniture du décor, des accessoires, etc. La municipalité et des bienfaiteurs patronnent ces entreprises, par l'intermédiaire d'une association, ou bien de clercs qui y apportent leur caution. Dans ce dernier cas, une interaction se met en place entre le responsable religieux du lieu, gardien de la tradition, et la troupe, puisque la pièce est montée sous son autorité religieuse. En tant que garant de la conformité du texte à la tradition, il est concerté sur tout changement envisagé ; s’il ne peut répondre, il en réfère à plus savant que lui, voire à son marja'. Bon nombre de clercs, aujourd'hui, soutiennent ce théâtre et y contribuent, parce qu'ils y voient une manière de transmettre le message de Husayn et de cultiver sa mémoire. D’autres se montrent indifférents et certains y sont hostiles ${ }^{17}$.

16 Les pièces n'obéissent pas à l'unité de temps ni de lieu. Les lieux scéniques sont juxtaposés, jalonnant l’itinéraire de Husayn, sa passion. L'accent est mis sur les paroles, prononcées avec emphase, et non sur le mouvement, ce qui 
quasi invariables, sont tirés d'ouvrages de référence qui constituent le corpus de la " tradition ${ }^{19}$ ».

17 À Nabatiyyeh, le texte de la pièce a été établi une première fois dans les années 1930 par le cheikh 'Abd al-Husayn Sâdiq ${ }^{20}$, assisté par des acteurs, mais le script varie sensiblement chaque année ${ }^{21}$. Dans les villages, les textes du Cortège des captives semblent provenir d'une même source puisqu'ils sont très proches (de même pour la mise en scène). Cette source pourrait être Majdal Silm² . L'enregistrement audio des représentations, puis aujourd'hui vidéo, permet de transcrire facilement le texte, et d'y apporter ensuite quelques changements. Hormis le script du drame de Karbala joué à Nabatiyyeh, qui fut publié dans des travaux de recherche ${ }^{23}$, les textes des pièces restent inédits, voire indisponibles. Dans un cas, chaque acteur n'a que le script du personnage qu'il incarne ; dans l'autre, le metteur en scène l'a écrit sur un carnet, à la main... On est bien loin des collections de manuscrits et d'imprimés auxquelles a donné lieu la ta'ziyeh persane.

18 Ce théâtre est un rituel, on l'a vu. Le public forme une Gemeinde, une communauté émotionnelle. Celle-ci s'émeut, pleure pendant la pièce ; elle n'applaudit pas à la fin. La tradition véhiculée est présentée comme immuable - ou bien ayant subi des altérations, des déformations, dont il faut se débarrasser - alors qu'elle est en perpétuel changement, renégociée chaque année. Dans l'interaction qui se joue alors, le religieux et le sacré s'accordent sur ce que l'on pourrait appeler le « noyau dur » du chiisme, à savoir le pacte des fidèles avec Husayn. Pratiquants rigoristes ou croyants laxistes peuvent se retrouver, là, pour ressouder la communauté. Ainsi, des fidèles « enreligiosés », pour traduire littéralement le terme courant de mutadayyîn, côtoient des croyants qui ont pris quelques distances avec la 
avant la représentation », se remémore l'un d'eux, qui jouait dans les années 1960.

\section{Politique et spectacle}

19 Ce théâtre rituel n'est pas coupé du contexte social, politique et culturel local, bien au contraire. C'est pourquoi, ces dernières années, il a connu des changements dans deux directions : vers la politisation, à l'instar des autres rituels observés pour Achoura, qui ont servi de plate-forme de mobilisation ; et vers la sécularisation, le spectacle, avec une certaine professionnalisation des troupes.

\section{Le public. Nabatiyyeh, février 2005.}

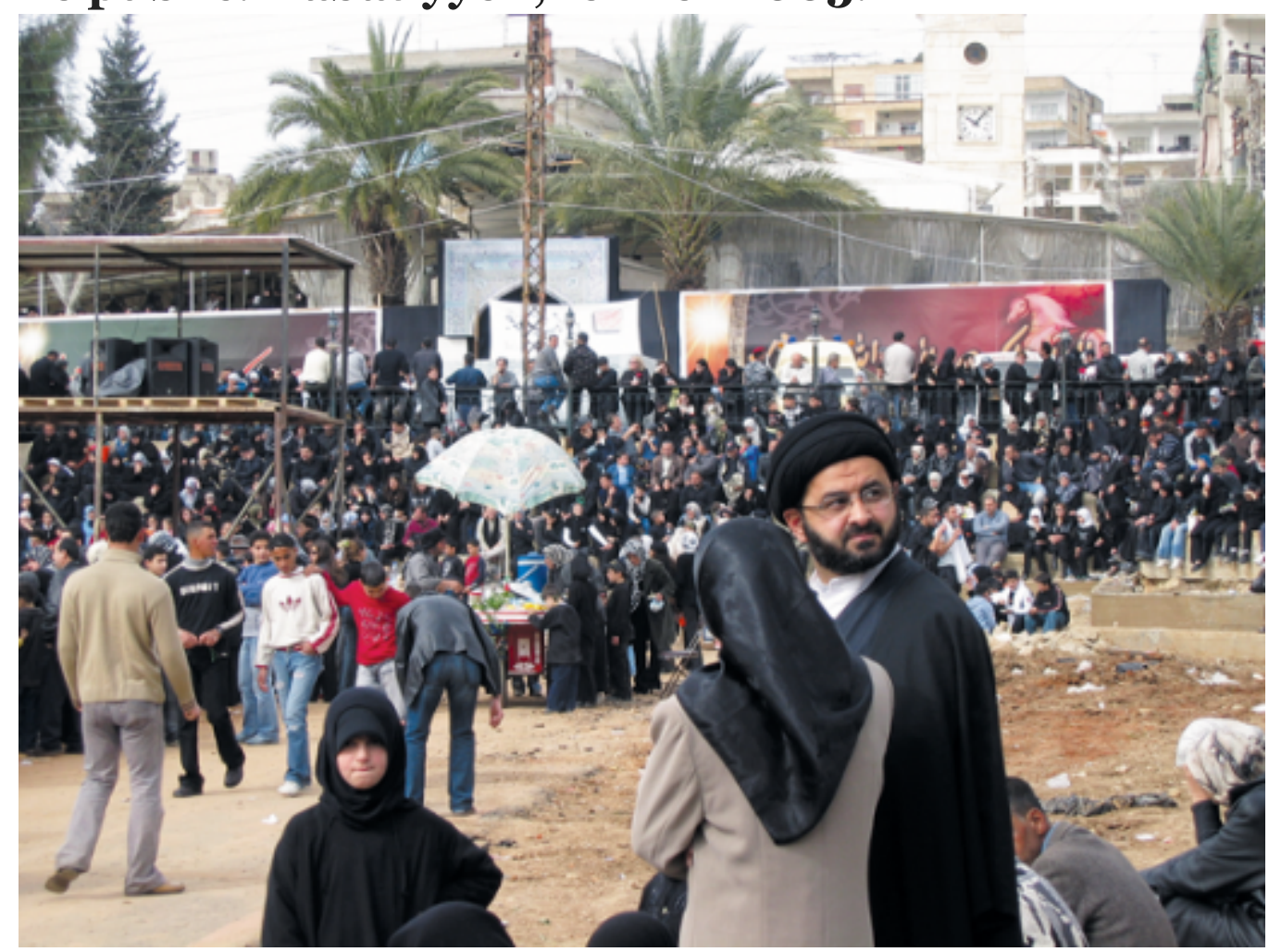

Photo S. Mervin.

20 Certes, les situations sont fort diverses, en fonction des villages. Il faudrait, en fait, pour chacun d'entre eux, écrire une histoire locale du théâtre rituel, couplée à une sociologie dec trounac Fn nutra una vive onnourranse c'overse entra

Ce site utilise des cookies et collecte des informations personnelles vous concernant. Pour plus de précisions, nous vous invitons à consulter notre politique de confidentialité (mise à jour le 25 juin 2018).

En poursuivant votre navigation, vous acceptez l'utilisation des cookies. 
message est vu différemment, surtout du point de vue politique.

21 Si la politique consiste à définir son ennemi, ce théâtre est pour cela un outil efficace. En Iran, à partir de l'époque safavide, le sultan calife ottoman a été comparé à Yazîd ; de même pour les souverains qajars quand les oulémas commencèrent à contester leur légitimité ${ }^{26}$. La politisation de la passion de Husayn et de son message n'est donc pas nouvelle. Toutefois, elle a été systématisée, au Liban-Sud, dans un processus qui se déroula en plusieurs étapes. La première commence à la fin des années 1950, avec une nouvelle donne politique et sociale au Liban-Sud : paupérisation de la région suite à la fermeture des frontières avec Israël ; émergence de groupes politiques indépendants de la za'âma locale (baathistes, communistes, etc.), due à la modernisation de l'enseignement. Le discours qui se met en place est que le combat de Husayn continue contre le tyran (associé à Yazîd) et contre le mal, quel qu'il soit (par exemple le sionisme $)^{27}$. Karbala est associée à la Palestine. Le poète Mahmoud Darwich n'a-t-il pas écrit : " Quand mes yeux te regardent fixement, je revois Karbala ${ }^{28} »$ ?

22 La deuxième étape est celle de l'ascension politique de Mûsâ Sadr qui, dans les années 1970, fut le premier religieux au Liban à recourir au message de Husayn pour mobiliser les foules. Avant lui, les oulémas chiites cautionnaient la za âma et la bourgeoisie locales qui, en retour, participaient à leur légitimation. Lui, appelle les chiites à relever la tête et à combattre pour leur liberté et leur indépendance, à l'exemple de l'imam, lors de rassemblements politiques qui se forment à l'occasion des célébrations de Achoura.

23 La troisième étape déterminante débute avec l'invasion israélienne, en 1982. Dans le village de Majdal Silm, deux hommes décident de monter des pièces pour célébrer 
demandais la 'abâya de mon grand-père, ma sœur prenait un mandîl... », se souvient-il. Khidr Wahab meurt dans une opération israélienne, dix ans plus tard. Depuis, Majdal Silm se contente d'une pièce, celle de la bataille de Karbala, jouée le jour de Achoura.

24 Il semble qu'il n'y avait plus, en 1974, que les représentations de Nabatiyyeh ${ }^{29}$. Après l'invasion israélienne, plusieurs pièces furent montées dans les villages, et celle-ci est un élément récurrent dans les récits de création ou de recréation d'un théâtre local. Bien plus, théâtre chiite et résistance sont liés. Ainsi, une altercation entre l'armée israélienne et la procession de pénitents qui défilaient à Nabatiyyeh, en 1983, est considérée comme le point de départ de la résistance au Liban-Sud. Les jeeps de l'armée tentèrent une percée dans la procession, pendant la représentation, et les pénitents réagirent en attaquant les jeeps, sabres à la main ; il y eut des morts de part et d'autre. "Ce fut la première étincelle qui déclencha la grande résistance contre l'occupation », déclara le cheikh 'Abd al-Husayn Sâdiq ${ }^{30}$. Un panneau, situé près du terrain où se donne la pièce, le rappelle.

25 La montée du Hezbollah et de l'idéologie de la résistance favorisa ce théâtre. Le Hezbollah, à la fin des années 1980, se mit à monter ses propres pièces. Lors de la représentation de la pièce que le parti monta pour l'arba în en 2006, il y eut peu de pleurs dans l'assistance, plutôt même de l'enthousiasme, des applaudissements. La politisation entraîne une manière de sécularisation - et la politique fait $\mathrm{du}$ rituel un spectacle. Soulignons, toutefois, que les frontières entre le religieux et le profane sont ici très ténues et que le spectacle est - forcément - une dimension intrinsèque de ce théâtre.

26 À la fin des années 1960, des «touristes » étaient signalés à Nabatiyyeh $^{31}$; on peut supposer qu'ils y venaient auparavant.

Ce site utilise des cookies et collecte des informations personnelles vous concernant. Pour plus de précisions, nous vous invitons à consulter notre politique de confidentialité (mise à jour le 25 juin 2018).

En poursuivant votre navigation, vous acceptez l'utilisation des cookies. 
ailleurs, n’hésite pas à inciter au renouvellement de la pièce, ce qui séduit la presse et suscite de nouveaux spectateurs.

Représentation du Cortège des captives. Kafr Kila, 2005.

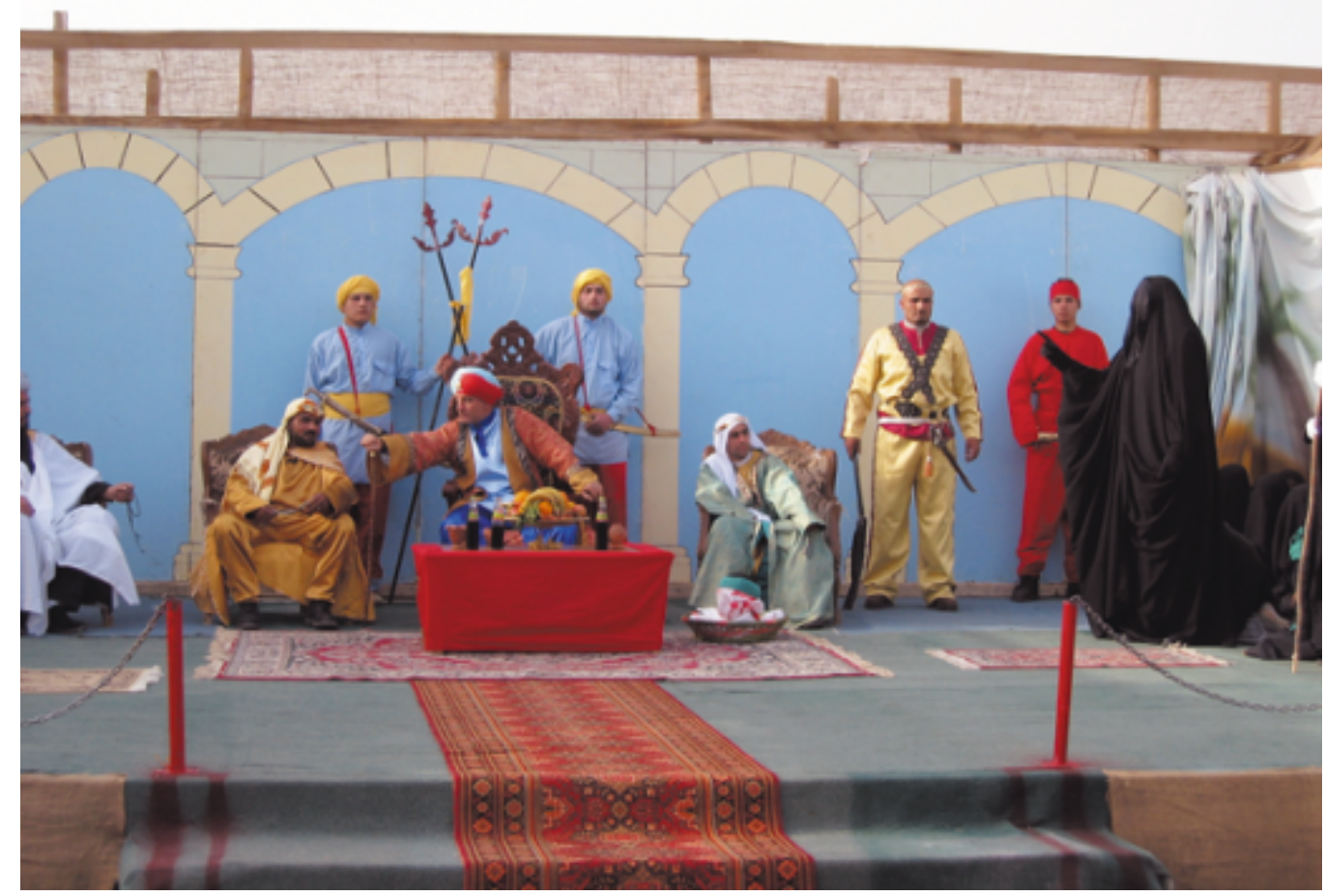

Photo S. Mervin.

27 En outre, aujourd'hui, certains villages ont tendance à ouvrir leurs représentations vers l'extérieur, s'adressant tant aux villages voisins qu'à d'autres communautés religieuses. Ainsi, en 2006, des chrétiens, des sunnites et des druzes assistèrent à la représentation du Cortège des captives, à Kafr Kila, selon son metteur en scène 'Alî Diya. En corrélation, le discours élaboré autour du message de Husayn se veut universaliste (kawnî), surtout s'il émane d'intellectuels de gauche convertis au théâtre religieux. Il vise à transcender le message, simple, véhiculé par les clercs qui s'attachent, eux, à l'authenticité et à la rigueur " historique ». Ainsi, Husayn est un paradigme où tous se retrouvent ; son exemplarité produit du sens aussi pour les « laïcisants » chiites, qui

Ce site utilise des cookies et collecte des informations personnelles vous concernant. Pour plus de précisions, nous vous invitons à consulter notre politique de confidentialité (mise à jour le 25 juin 2018).

En poursuivant votre navigation, vous acceptez l'utilisation des cookies. 
Kafr Kila ou dans la banlieue sud. Dans les deux premiers cas, on recrute des acteurs de théâtre qui sont populaires parce que, faute de gagner leur vie dans cette seule branche, ils jouent aussi dans les feuilletons ou des films télévisés. Ils sont donc extérieurs à la communauté devant laquelle ils se produisent, mais lui sont familiers. C'est le cas de 'Ammâr Chalaq, qui joua le rôle de Husayn à Nabatiyyeh, en 2006. Ces acteurs professionnels sont donc rémunérés et côtoient des acteurs locaux, amateurs, qui ne le sont pas. Ceux-ci jouent parfois le même rôle pendant des années : ainsi d'Abû Racha, qui incarne le personnage de Chemr $^{32}$ depuis cinquante-quatre ans, ressortant chaque année son costume. Ces acteurs locaux connaissent leur rôle, mais aussi tout le script de la pièce ainsi que l'histoire des représentations, dont ils sont la mémoire. Ils sont des références que l'on consulte et des personnalités locales. Personne n'oserait leur enlever leur rôle. Ainsi, en 2006, le personnage de Chemr devait apparaître à cheval, et Abû Racha était trop âgé pour le faire : il fut doublé pour ces scènes et assura le reste du rôle.

29 À Kafr Kila, depuis plusieurs années, un acteur professionnel, Khâlid al-Sayyid, vient de Beyrouth pour jouer le rôle de l'émissaire du roi chrétien qui rend visite à Yazîd et s'offusque de voir, à ses pieds, la tête de Husayn. Comme à Nabatiyyeh, le recours à des acteurs professionnels résulte de la professionnalisation des metteurs en scène. À Kafr Kila, 'Alî Diya est un amateur éclairé qui consacre un temps croissant à ses projets de théâtre ; pour certains d'entre eux, il travaille en collaboration avec Husâm Sabbâh, un professionnel. En 2006, ce dernier a monté la pièce de Nabatiyyeh, dont il est originaire. Avant lui, le cheikh l'avait confiée à d'autres metteurs en scène reconnus, mais « étrangers » à la communauté locale et au chiisme, Ra'îf 
30 Un tournant a été marqué par la prestation de Ra’̂̂f Karam, un metteur en scène qui s'était déjà illustré dans le théâtre de rue à grand spectacle, dans les années 1980, après avoir soutenu en France une thèse de doctorat sur Brecht et Artaud $^{34}$. Il s'est lancé dans cette entreprise, « au seuil du théâtre et du réel, du sacré et du profane », en poursuivant une réflexion déjà entamée sur l'idée de seuil, à la fois intérieur et extérieur. Il l'intégra dans un concept théâtral de son invention, celui de " théâtre catastrophique (kawârithî) ». L'expérience du drame de Karbala l’intéressait d'autant plus qu'il voyait en Achoura un concept universel. "Husayn m'intéresse comme le Christ, comme Guevara et tous les martyrs du Liban », explique-t-il. Ra'îf Karam a introduit des changements dans le script de la pièce (par exemple, il fait apparaître Yazîd) et dans la mise en scène proprement dite. Ainsi, il a fait appel à un groupe de musiciens marocains pour composer la bande-son de la pièce ; il a introduit un chœur de femmes - dont la performance fut uniquement gestuelle ; il a engagé un directeur artistique qui a suspendu une toile, au fond du terrain, laquelle se recouvrit progressivement d'une couleur rose foncé, symbolisant le martyre, durant le déroulement du drame. " J'ai tenté de ramasser tous les éléments traditionnels dans un tunnel très postmoderne », conclut le metteur en scène ${ }^{35}$.

31 L'année suivante, Mashhûr Mustafâ a poursuivi la réflexion en direction de la modernisation et du symbolisme en développant une autre idée : à la fin du drame, les figurantes qui incarnaient les femmes de la famille de Husayn se mirent en cercle pour tendre un immense tissu rouge symbolisant, lui aussi, le sang des martyrs. Sans doute dans un souci de professionnalisation, en 2006, le cheikh 'Abd al-Husayn Sâdiq demanda la construction d'une nouvelle scène : 
nuançant le symbolisme des couleurs utilisées, et une bandeson à base de flûte (nay) et de voix fredonnées fut composée. Certains metteurs en scènes s'emploient donc à apporter peu à peu ce qu'ils considèrent comme des améliorations à ce théâtre qui, s'il est sacré, est aussi populaire et quelque peu «bricolé ».

32 Certains villages refusent cette tendance à la professionnalisation : on reste entre soi (amateurs, habitants du village). C'est le cas à Majdal Silm, où le théâtre est une affaire de famille. Les répétitions se font dans ce cadre, et non dans celui, plus formel, de réunions de la troupe ; les enfants, en grandissant, changent de rôle. En outre, les organisateurs sont rétifs à la médiatisation des représentations : ils ne fabriquent pas de CD Roms des pièces, comme il en existe ailleurs, qui se vendent dans les rues, le jour de Achoura. Ils s'ouvrent volontiers à l'extérieur, mais aux villages alentours, ou bien à un public motivé, qui partage leurs aspirations, comme celui qui vient, depuis quelques années, de la Bekaa.

\section{Deux pièces, deux visions du monde}

33 Un pas de plus vers la modernité théâtrale a été franchi par Husâm Sabbâh, qui tenta une expérience nouvelle en présentant une pièce intitulée al-Husayn shâhidan (Husayn témoin), au centre culturel Nabîh Berrî, près de Nabatiyyeh. La date choisie fut le 6 mai 2005, qui ne correspondait pas à une célébration religieuse, mais au « Jour des martyrs » au Liban. Après une série d'hommages au Premier ministre assassiné, Rafic Hariri, un film visant à faire apparaître le sens du martyre de Husayn et des récitals de poésie à la gloire de l'imam, la pièce fut jouée. C'est une sîra de Husayn, le récit de sa vie mis en scène avec le souci de restituer l'essentiel de son message. La pièce prend la forme d'une

Ce site utilise des cookies et collecte des informations personnelles vous concernant. Pour plus de précisions, nous vous invitons à consulter notre politique de confidentialité (mise à jour le 25 juin 2018).

En poursuivant votre navigation, vous acceptez l'utilisation des cookies. 
associées au noir et au blanc. Le scénographe - un professionnel - a représenté le campement de Husayn par un escalier et quatre petites tentures, au second plan. Le clou de la représentation est la bataille de Karbala qui est non plus mimée, mais dansée par une troupe dont les mouvements ont été savamment mis en scène par une chorégraphe. Pas de sang, même suggéré. L'actrice qui joue Zaynab vient ramasser les vêtements noirs tombés à terre, qui symbolisent les martyrs, et les empile dans ses bras.

34 Dans la salle, au premier rang, quelques clercs chiites - et un grec-orthodoxe - apportèrent leur caution à cette expérience hardie, qui ne manqua pas de provoquer son lot de critiques. Les organisateurs auraient souhaité donner d'autres représentations, mais il leur fut difficile de trouver un cadre. En effet, si leur approche est résolument moderne, ils ont des difficultés à faire admettre le caractère universel de leur théâtre, qui reste un " théâtre chiite ».

35 Une autre approche de la modernité est promue par le Hezbollah, qui, dit-on au bureau de la production culturelle, ne fait pas « de l'art pour l'art », mais met la forme au service d'un contenu à la fois religieux et politique. Il s'agit de montrer que la bataille de Karbala n'est pas seulement un événement historique, mais qu'elle peut se reproduire n'importe quand, et de « relier Husayn à la résistance ». Les pièces de théâtre sont vues comme des « écoles politiques », où l'on parle de Achoura avec un nouveau langage, conçu par des professionnels ${ }^{36}$. De même, le Hezbollah a monté des panoramas, à Beyrouth, puis à Tyr, et a organisé des expositions de dessins ; dans les villages, les scouts du parti (Kashshâf al-imâm al-Mahdî) s'en chargent.

36 En 2006, le bureau de production culturelle a supervisé le montage d'une pièce qui fut jouée deux fois par jour, durant la semaine précédant l'arba‘în, au sous-sol d'une mosquée 
calendrier chrétien] ». Le ton est donné. Tout le principe repose sur l'alternance ou la présence simultanée d'images filmées et de jeu théâtral et sur le passage du passé au présent. Les images, au début, montrent la route de Karbala, une cavalcade à cheval dans le désert... Sur scène, c'est le campement de Husayn. Les décors et les costumes sont réalisés dans de bons matériaux, ce qui laisse deviner les moyens mis en œuvre. Puis l'on arrive à la bataille qui se déroule à la fois sur l'écran de fond et sur la scène. Après le martyre de l'imam, il reste son cheval blanc : c'est une image connue, qui rappelle le tableau d'un peintre iranien. Une fumée envahit la scène : le campement a été incendié. Soudain vrombit le bruit d'un moteur d'hélicoptère qui nous transporte au $\mathrm{xx}^{\mathrm{e}}$ siècle. C'est l'invasion israélienne. Puis une succession d'images raconte l'histoire de la résistance islamique, avec les leaders du parti (Khomeini, Râghib Harb, 'Abbâs Musâwî...), des opérations militaires, des combattants sur le départ ou faisant leur testament, des blessés et des armes. Sur scène, voici Zaynab disant son discours. Et l'on revient à la période contemporaine, avec des images de la libération du Liban-Sud en 2000, de colonies israéliennes, de Jérusalem, d'opérations... Après ces aller et retour construits sur un rythme serré, le mot de la fin est celui que prononce Zaynab devant Yazîd, à Damas, son fameux discours qui se termine par «Vous n'effacerez pas notre mémoire... » (lan tamhû dhikrânâ). C'est le titre de la pièce.

\section{Bibliographie}

Des DOI sont automatiquement ajoutés aux références par Bilbo, l'outil d'annotation bibliographique d'OpenEdition.

Les utilisateurs des institutions qui sont abonnées à un des programmes freemium d'OpenEdition peuvent télécharger les références bibliographiques pour lequelles Bilbo a trouvé

Ce site utilise des cookies et collecte des informations personnelles vous concernant. Pour plus de précisions, nous vous invitons à consulter notre politique de confidentialité (mise à jour le 25 juin 2018).

En poursuivant votre navigation, vous acceptez l'utilisation des cookies. 
d'OpenEdition.

Si vous souhaitez que votre institution souscrive à l'un des programmes freemium d'OpenEdition et bénéficie de ses services, écrivez à : contact@openedition.org

Format

APA

MLA

Chicago

Le service d'export bibliographique est disponible aux institutions qui ont souscrit à un des programmes freemium d'OpenEdition.

Si vous souhaitez que votre institution souscrive à l'un des programmes freemium d'OpenEdition et bénéficie de ses services, écrivez à : contact@openedition.org

Ansari Pettys R., 1981 : "The Ta'zieh: Ritual Enactment of Persian Renewal », Theatre Journal, 33/3, oct., p. 341-354.

DOI : 10.2307/3207032

Ayoub M., 1978 : A Redemptive Suffering in Islam. A Study of the Devotional Aspects of 'Ashûrâ in Twelver Shî‘ism,La Hague-Paris, Mouton.

Calmard J., 1974 : « Le mécénat des représentations de ta'ziye : I. Les précurseurs de Nâseroddin Châh », dans Le monde iranien et l'islam, t. II, Genève-Paris, Droz, p. 73-126.

Calmard J., 1975 : Le culte de l'imâm Husayn. Étude sur la commémoration du drame de Karbala dans l'Iran présafavide, thèse de $3^{\mathrm{e}}$ cycle, 2 vol., Paris, EPHE.

CALMard J., 1976-1977 : « Le mécénat des représentations de ta'ziyé : II. Les débuts de Nâseroddin Châh », dans Le monde iranian at lisclam + IV Conòva-Paric Nroz n 100-16o

Ce site utilise des cookies et collecte des informations personnelles vous concernant. Pour plus de précisions, nous vous invitons à consulter notre politique de confidentialité (mise à jour le 25 juin 2018).

En poursuivant votre navigation, vous acceptez l'utilisation des cookies. 
Cerulli E., 1970 : « Le théâtre persan », dans T. Fahd (éd.), Le shi'isme imâmite, Colloque de Strasbourg, Paris, Brunschvig, PUF, p. 281-194.

Chelkowski P. J. (éd.), 1979 : Ta'ziyeh: Ritual and Drama in Iran, New York, New York University Press.

Format

APA

MLA

Chicago

Le service d'export bibliographique est disponible aux institutions qui ont souscrit à un des programmes freemium d'OpenEdition.

Si vous souhaitez que votre institution souscrive à l'un des programmes freemium d'OpenEdition et bénéficie de ses services, écrivez à : contact@openedition.org

Chelkowski P. J., 1985 : " Shia Muslim Processional Performances ", The Drama Review,29/3, automne 1985, p. 18-30.

DOI : $10.2307 / 1145650$

Chelkowski P. J. (dir.), 2005 : « From Karbala to new York: Ta'ziyeh on the Move », The Drama Review, 49/4, hiver 2005.

Chrara W., 1968 : Transformation d'une manifestation religieuse dans un village du Liban-sud (Ashura), Beyrouth, Publications du Centre de recherches de l'Université libanaise.

Colloque «Ashura », Cahiers de l'École supérieure des Lettres, 5 (1974), Beyrouth.

Ce site utilise des cookies et collecte des informations personnelles vous concernant. Pour plus de précisions, nous vous invitons à consulter notre politique de confidentialité (mise à jour le 25 juin 2018).

En poursuivant votre navigation, vous acceptez l'utilisation des cookies. 
Gaffary F., 1984 : " Evolution of Rituals and Theater in Iran », Iranian Studies, 4, p. 361-389.

Gobineau (comte de), 1865 : Les religions et les philosophies dans l'Asie centrale, Paris, Didier et Cie Librairie académique, $2^{\mathrm{e}}$ éd.

Goody J., 2003 : La peur des représentations. L’ambivalence à l'égard des images, du théâtre, de la fiction, des reliques et de la sexualité, Paris, La Découverte.

Haydari I., 1977 : « Die Ta'ziya, das schiitische Passionsspiel im Libanon », ZDMG, sup. III, p. 430-437.

HAydari I., 2002 : " The Rituals of 'Ashura: Genealogy, Functions, Actors and Structures ", dans F. Abdul-Jabar (éd.), Ayatollahs, Sufis and Ideologues. State, Religion and Social Movements in Iraq, Saqi Books, p. 101-113.

JABER T., 1980 : Chiites et pouvoir politique au Liban (19671974), thèse de doctorat de $3^{\mathrm{e}}$ cycle, université de Paris 7.

Format

APA

MLA

Chicago

Le service d'export bibliographique est disponible aux institutions qui ont souscrit à un des programmes freemium d'OpenEdition.

Si vous souhaitez que votre institution souscrive à l'un des programmes freemium d'OpenEdition et bénéficie de ses services, écrivez à : contact@openedition.org

Karam R., 2003 : " The Taste of Bread. My Theatrical Experience in Lebanon », The Drama Review, 44/4, hiver

Ce site utilise des cookies et collecte des informations personnelles vous concernant. Pour plus de précisions, nous vous invitons à consulter notre politique de confidentialité (mise à jour le 25 juin 2018). 
MaAtouk Fr., 1974b : " Analyse dramaturgique de "Zain el 'Abidine" »,dans Colloque « Ashura », Cahiers de l'Ecole Supérieure des Lettres.

Malekpour J., 2004 : The Islamic Drama, Londres, Franck Cass.

Mervin S., 2000 : Un réformisme chiite. Ulémas et lettrés du Jabal 'Âmil (actuel Liban-Sud) de la fin de l'Empire ottoman à l'indépendance du Liban, Paris, KarthalaCERMOC-IFEAD.

Format

APA

MLA

Chicago

Le service d'export bibliographique est disponible aux institutions qui ont souscrit à un des programmes freemium d'OpenEdition.

Si vous souhaitez que votre institution souscrive à l'un des programmes freemium d'OpenEdition et bénéficie de ses services, écrivez à : contact@openedition.org

Mervin S., 2006 : « Les larmes et le sang des chiites : corps et pratiques rituelles lors des célébrations de 'âshûrâ' ", REMM,113-114, Le corps et le sacré dans l'Orient musulman, nov., p. 153-166.

DOI : $10.4000 / \mathrm{remmm} .2973$

NAJAH Sl., 1974 : La célébration de la 'Ashura à Nabatiyye, Liban,thèse de $3^{\mathrm{e}}$ cycle, Université de Provence, Aix-enProvence, 2 vol.

Nakkash Y., 1993 : " An Attempt to Trace the Origin of the Rituals of 'Ashûrâ' », DieWelt des Islams, 33, p. 161-181.

Ce site utilise des cookies et collecte des informations personnelles vous concernant. Pour plus de précisions, nous vous invitons à consulter notre politique de confidentialité (mise à jour le 25 juin 2018).

En poursuivant votre navigation, vous acceptez l'utilisation des cookies.

\section{Fermer}


Norton R. A., 2005 : «Ritual, Blood, and Shiite Identity », The Drama Review, 49/4, hiver 2005, p. 140-155.

RizkALlaH R., 1977 : Contribution à une approche psychosociologique d'un rite chez les chiites du Liban sud ('Âshûrâ'), thèse de $3^{\mathrm{e}}$ cycle, Paris 7.

Shams AL-Din, shaykh Muhammed Mehdi, 1985 : The Rising of al Husayn. Its Impact on the Consciousness of Muslim society, Londres, Muhammadi Trust.

Virolleaud Ch., 1950 : Le théâtre persan ou le drame de Kerbéla, Paris, A. Maisonneuve, 141 p.

\section{Notes}

1. Pour une vision contemporaine de la signification du martyre de Husayn, cf. Shams AL-Din1985, chap. 1. Pour une analyse, voir Ayoub 1978.

2. Virolleaud 1950, p. 6.

3. Sur l'histoire de ces rituels, voir NAKKASH 1993.

4. Le tatbîr consiste à se frapper le crâne, préalablement entaillé, avec le plat de la main ou de la lame d'un sabre, à un rythme régulier. Pour une description plus détaillée de ces rituels, cf. Mervin 2006. Sur les séances de déploration, cf. DeEв 2006, chap. 4.

5. Cerulli 1970, p. 286-287. On trouve aujourd'hui des textes de pièces iraniennes sur www.iranian.com.

6. Les observations notées dans cet article sont le fruit d'enquêtes de terrain effectuées au Liban entre 2004 et 2006.

7. On citera deux ouvrages récents, celui de W. Floor (2005), et celui de J. Malekpour (2004). À cela, il faut ajouter les travaux fondateurs de P. Chelkowski (1979) et, récemment, le numéro de la revue The Drama Review qu'il a dirigé (ChelKowski 2005).

8. Sur ces questions, voir les travaux de J. Calmard (1974, 1975, 1976-

Ce site utilise des cookies et collecte des informations personnelles vous concernant. Pour plus de précisions, nous vous invitons à consulter notre politique de confidentialité (mise à jour le 25 juin 2018).

En poursuivant votre navigation, vous acceptez l'utilisation des cookies.

\section{Fermer}


11. Pour une histoire détaillée de l'implantation de ce rituel, cf. Mervin 2000, p. 245 sqq.

12. Al-Hurr était un soldat de l'armée omeyyade qui se rallia à Husayn, combattit à ses côtés, et périt dans la bataille. Cf. le récit de son « retournement » dans le texte publié par Fr. MaAtouk (1974a), p. 158-159.

13. En 1974, Fr. Maatouk affirme qu'il n'y eut qu'une brève apparition féminine parmi les acteurs, en 1933. Cf. MaAtouk 1974a, p. 46.

14. Lieu de culte spécifique au chiisme où se tiennent des séances de déploration.

15. Entretien avec des acteurs, Majdal Silm, 05/02/2006.

16. Entretiens avec des membres de la troupe de Kafr Kila, juin 2005, pour un film documentaire réalisé sur ce sujet, Le cortège des captives (tragédie chiite).

17. Ainsi de 'Abd al-Amîr Qabalân, président du Conseil supérieur chiite, qui n’approuve pas les représentations. NorTon 2005, p. 152.

18. Chrara 1968, p. 54

19. Il s'agit des ouvrages classiques de maqtal (récit de martyre, ici, celui de Husayn), ou bien d'ouvrages plus contemporains, composés pour les récitants de séances de déploration.

20. 'Abd al-Husayn Sâdiq exerça son magistère à Nabatiyyeh de 1906 à sa mort, en 1942. Son petit-fils, qui porte le même nom, est aujourd'hui l’imam de la ville et supervise le montage de la pièce.

21. NoRTON 2005, p. 151.

22. Des membres de la troupe de Majdal racontent avoir assisté à une pièce calquée sur la leur, à tel point qu'y était fidèlement reproduite... une toux dont avait été prise l'actrice jouant Zaynab pendant une représentation!

23. MAatouk 1974a, p. 175-195 ; JABER 1980, p. 341-348.

24. Cf. Chrara 1968, p. 35-36.

Ce site utilise des cookies et collecte des informations personnelles vous concernant. Pour plus de précisions, nous vous invitons à consulter notre politique de confidentialité (mise à jour le 25 juin 2018).

En poursuivant votre navigation, vous acceptez l'utilisation des cookies. 
29. On y jouait alors Le martyre de l’imam Husayn et Zayn al-‘̂̉bidîn à Damas, cf. MaAtouk 1974a et 1974b ; W. Chrara présente, lui aussi, le cas de Nabatiyyeh comme isolé, cf. Chrara 1968, p. 43. Néanmoins, il est possible que ces deux auteurs n'aient pas eu connaissance d'autres pièces, ailleurs. Aujourd'hui, ce théâtre reste largement ignoré en dehors des villages où il est joué.

30. 'Alî Dâwûd, "'Âchûrâ' 1983 malhama butûliyya... ", al-Liwâ, 06/05/1998.

31. Chrara 1968, p. 34.

32. Chemr/Chamîr est le meurtrier de Husayn. Il est donc, avec Yazîd, l'archétype du « méchant ».

33. Selon Ra’îf Karam, le marja“ 'Ali Sistani, sollicité à ce sujet, a émis une fatwâ qui ne le permettait pas.

34. Sur la trajectoire de Ra'îf Karam, voir KARAM 2003.

35. Entretien avec Ra'îf Karam, Beyrouth, mars 2005. Sur son expérience à Nabatiyyeh, voir aussi son interview dans al-Safîr, 24/01/2004 et l'article de Abbas Beydoun, « al-usbû' al-damawî... 'âchûrâ' Ra'îf Karam wa Mîl Gîbsûn », al-Safîr, 12/03/2004 : le journaliste y établit un parallèle entre la pièce mise en scène par R. Karam et le film réalisé par Mel Gibson sur la passion du Christ.

36. Entretien avec un responsable du bureau, Muhammad Kawtharânî, Hârat Hurayk, 29/03/2006.

\section{Auteur}

\section{Sabrina Mervin}

\section{Chargée de recherche au CNRS et chercheur à l'Institut français du Proche-Orient (IFPO, Beyrouth)}

(C) Presses de l’Ifpo, 2007

Ce site utilise des cookies et collecte des informations personnelles vous concernant. Pour plus de précisions, nous vous invitons à consulter notre politique de confidentialité (mise à jour le 25 juin 2018).

En poursuivant votre navigation, vous acceptez l'utilisation des cookies. 
Disponible sur Internet : <http://books.openedition.org/ifpo/546>. ISBN : 9782351592687. DOI : 10.4000/books.ifpo.546.

\section{Référence électronique du livre}

PUIG, Nicolas (dir.) ; MERMIER, Franck (dir.). Itinéraires esthétiques et scènes culturelles au Proche-Orient. Nouvelle édition [en ligne]. Beyrouth : Presses de l'Ifpo, 2007 (généré le 27 août 2018). Disponible sur Internet : <http://books.openedition.org/ifpo/517>. ISBN : 9782351592687. DOI : 10.400o/books.ifpo.517.

Compatible avec Zotero 